\title{
Semiclassical extension of the Landau-Teller theory of collisional energy transfer
}

\author{
E. I. Dashevskaya \\ Department of Chemistry, Technion-Israel Institute of Technology, Haifa 32000, Israel and \\ Max-Planck-Institut für Biophysikalische Chemie, Am Fassberg, D-37077 Göttingen, Germany \\ I. Litvin \\ Department of Chemistry, Technion-Israel Institute of Technology, Haifa 32000, Israel and Institut für \\ Physikalische Chemie, Universität Göttingen, Tammannstrasse 6, D-37077 Göttingen, Germany \\ E. E. Nikitin \\ Department of Chemistry, Technion-Israel Institute of Technology, Haifa 32000, Israel and \\ Max-Planck-Institut für Biophysikalische Chemie, Am Fassberg, D-37077 Göttingen, Germany \\ J. Troe ${ }^{a)}$ \\ Max-Planck-Institut für Biophysikalische Chemie, Am Fassberg, D-37077 Göttingen, Germany and Institut \\ für Physikalische Chemie, Universität Göttingen, Tammannstrasse 6, D-37077 Göttingen, Germany
}

(Received 20 March 2006; accepted 1 September 2006; published online 20 October 2006)

A semiclassical version of the quantum coupled-states approximation for the vibrational relaxation of diatomic molecules in collisions with monatomic bath gases is presented. It is based on the effective mass approximation and a recovery of the semiclassical Landau exponent from the classical Landau-Teller collision time. For an interaction with small anisotropy, the Landau exponent includes first order corrections with respect to the orientational dependence of the collision time and the effective mass. The relaxation $\mathrm{N}_{2}(v=1) \rightarrow \mathrm{N}_{2}(v=0)$ in He is discussed as an example. Employing the available vibrationally elastic potential, the semiclassical approach describes the temperature dependence of the rate constant $k_{10}(T)$ over seven orders of magnitude across the temperature range of 70-3000 $\mathrm{K}$ in agreement with experimental data and quantum coupled-states calculations. For this system, the hierarchy of corrections to the Landau-Teller conventional treatment in the order of importance is the following: quantum effects in the energy release, dynamical contributions of the rotation of $\mathrm{N}_{2}$ to the vibrational transition, and deviations of the interaction potential from a purely repulsive form. The described treatment provides significant simplifications over complete coupled-states calculations such that applications to more complex situations appear promising. (c) 2006 American Institute of Physics. [DOI: 10.1063/1.2357951]

\section{INTRODUCTION}

The accurate calculation of rate coefficients for collision-induced transitions between the vibrational states of molecules in a gaseous heat bath requires the solution of quantum scattering equations for a set of potential energy surfaces (PESs). If both the molecule and the collision partner are in a closed electronic shell, one needs only one PES, the adiabatic intra- and intermolecular potentials in a given electronic state. For the special case of a diatomic molecule in a monatomic bath gas, the appropriate PES, $U(R, \gamma, r)$, depends on three coordinates, the interfragment distance $R$, the intramolecular distance $r$, and the angle $\gamma$ between the vectors $\mathbf{R}$ and $\mathbf{r}$. For such systems, the scattering equations have been investigated in detail and a number of approximate treatments were suggested. Optimal results were obtained with the close-coupling (CC) method, which numerically solves the system of scattering equations in the adopted basis set of wave functions. A widely used approximation to the CC method, which markedly simplifies the structure of

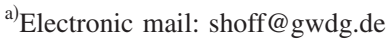

the equations, is the coupled-states (CS) approximation. This approximation assumes the conservation of the projection of the intrinsic angular momentum vector of the diatom onto $\mathbf{R}$ during the whole collision event and takes into account, though in a limited way, the strong coupling between the translational and the rotational motion. Normally, the CC and CS approximations account for the coupling between several vibrational states of the diatom [the so-called vibrational close coupling (VCC)].

An alternative to the full quantum treatment is provided by the full classical treatment with initial quantum conditions for the vibrational and rotational action variables, the socalled quasiclassical trajectory (QCT) method. Within this approach, the transition probabilities are determined by the number of trajectories that fall into bins for final action variables; these contiguous bins are centered at the quantum values of the action variables. The advantage of QCT method over quantum scattering approaches is that it can be applied, with reasonable computational efforts, to systems with many degrees of freedom, such as the collisions of triatomic and diatomic molecules, such as demonstrated, e.g., in Refs. 2 and 3. The disadvantage is that the bins should be suffi- 
ciently filled in order to calculate the well-defined probabilities. If some probabilities are small enough, the respective bins turn out to be empty (the so-called classically forbidden transitions), implying complete breakdown of the QCT method and calling for a more sophisticated semiclassical methods. ${ }^{4}$ For the vibrational transitions in atom-diatom collisions, the QCT method fails under near adiabatic conditions when the vibrational period is much shorter then the appropriate collision time.

Still another way to attack the problem with classically forbidden transitions is to describe the relaxing mode quantum mechanically and the other degree of freedom classically. ${ }^{5}$ If within this approach one takes advantage of two important features of vibrationally inelastic collisions, namely the weak coupling of the vibrational mode to the heat-bath modes for transitions between low-lying vibrational states and the quasiclassical character of the heat-bath modes, one can use the general formalism of the linear response theory. ${ }^{6}$ The model of Landau and Teller ${ }^{7}$ can be considered as one of the first examples of this approach that shows the difficult consequences of splitting the whole system into quantum and classical subsystems. In short, when the quantum of vibrational energy $\Delta E$ is released into the heat bath of temperature $T$ under the condition that the former is comparable with or larger than $k T$, it produces a strong local perturbation in the bath modes irrespective of the coupling strength between the vibrational and the bath degrees of freedom. A classical correlation function of the coupling interaction fails to account for this effect, and, as a result, the straightforward application of the respective linear response theory for calculation of the state-to-state rate coefficients for collision-induced vibrational transitions, $k_{n, n^{\prime}}(T)$ will lead to the expressions that disagree with the detailed balance relation for $n \rightarrow n^{\prime}$ and $n^{\prime} \rightarrow n$ transitions. There exists an extensive body of work that aims at correcting the classical correlation function in such a way as to ensure the correct detailed balance behavior. ${ }^{8-15}$ We also have recently discussed this problem ${ }^{16}$ and suggested the way to restore the detailed balance relation by using the semiclassical (SC) approximation as described in the Landau and Lifshitz's textbook ${ }^{17}$ where it is called the quasiclassical approximation. The latter is based on the extension of the WKB approximation for the wave functions of the bath modes, and it is different in spirit from the QCT method mentioned above.

The aim of the present article is to generalize the results of our previous paper ${ }^{16}$ and determine the hierarchy of the corrections to the original Landau-Teller (LT) model. We do this by providing a SC bridge between the LT treatment and accurate (or approximate) quantum calculations. For this purpose we adopt Landau's SC approximation ${ }^{18}$ in its more recent formulation, ${ }^{19}$ which allows us to relate Landau's quasiclassical exponential factor of the rate coefficient with the classical LT collision time. This procedure identifies the dominant part of the temperature dependence of $k_{10}(T)$ without performing quasiclassical calculations explicitly. It should be noted that the determination of the temperaturedependent factor is easier than the calculation of the absolute value of the rate coefficient since the former is determined by the interaction potential between the atom and the nonvibrating diatom only. The calculation of the absolute value in addition requires information on the vibration-rotationtranslation coupling and often necessitates an approach which goes beyond the first-order approximation. ${ }^{20-23}$ The described approach can also be applied to more complicated situations such as the vibrational relaxation of diatoms in compressed gases and liquids where a full quantum treatment appears not feasible yet.

\section{BASIC ASSUMPTIONS OF THE LANDAU-TELLER MODEL}

The basic assumptions of the LT model of collisional energy transfer ${ }^{1}$ have been discussed recently again. ${ }^{16}$ In order to be specific, in the following we formulate them for a $1 \rightarrow 0$ vibrational transition. The assumptions are as follows:

(i) a collinear collision of a heat-bath atom $\mathrm{A}$ with a diatom $\mathrm{BC}$;

(ii) weak coupling of the vibration of the diatom to the relative translation, being proportional to the vibrational amplitude;

(iii) neglect of the influence of the released energy on the relative motion of the collision partners [the so-called common trajectory (CT) or external field approximation]. Within this approximation, the initial $\left(E_{i}\right)$ and the final $\left(E_{f}\right)$ collision energies are considered to be close to each other and approximately equal to a certain average energy $E$; and

(iv) near-adiabatic conditions for the collision, which leads to an exponential dependence of the transition probability $P_{10}$ on the vibrational frequency $\omega$.

Under the described conditions, the the transition probability follows as

$P_{10}(E)=A \exp (-2 \omega \tau(E))$,

where $\tau(E)$ is the LT collision time and $\hbar \omega$ is the vibrational quantum. One assumes that $\omega \tau(E) \gg 1$ and that the preexponential factor $A$ has only a weak energy dependence in comparison to that of the exponential factor. The collision time $\tau(E)$, for an arbitrary potential $U(R)$, can be calculated as

$$
\tau(E)=\int_{R_{s}}^{R_{t}} \frac{\sqrt{2 \mu} d R}{\sqrt{U(R)-E}}
$$

where $R_{t}$ is the A-B distance at the turning point of the motion and $R_{s}<R_{t}$ is the distance $R$ at which the integral can be considered as converged. For a repulsive interaction between $\mathrm{A}$ and $\mathrm{B}$ of the exponential form, $U\left(R_{\mathrm{AB}}\right) \propto \exp \left(-R_{\mathrm{AB}} / a\right)$, the LT collision time is given by $\tau(E) \equiv \tau^{\mathrm{LT}}(E)=\pi a \sqrt{2 \mu} / \sqrt{E}$, where $\mu$ is the reduced mass of $\mathrm{A}$ and $\mathrm{BC}$.

Under the condition $\omega \tau \gg 1$, the temperature dependence of the rate coefficient $k_{10}(T)$ is governed essentially by the exponential factor in the transition probability. The thermal averaging over the collision energies can be performed in the steepest descent approximation yielding 


$$
k_{10}(T) \propto \exp \left(-2 \omega \tau\left(E^{*}\right)-E^{*} / k T\right),
$$

where $k$ is the Boltzmann constant. Here the temperaturedependent energy of the steepest descent point $E^{*}$ is found from the equation

$$
2 \omega d \tau\left(E^{*}\right) / d E^{*}+1 / k T=0 .
$$

For the LT collision time, Eq. (3) then assumes the form

$$
k_{10}^{\mathrm{LT}}(T) \propto \exp \left(-3\left(T^{\mathrm{LT}} / T\right)^{1 / 3}\right),
$$

with $T^{\mathrm{LT}}=\pi^{2} \omega^{2} a^{2} \mu / 2 k$. Equation (5) gives the linear dependence of $\ln k_{10}^{\mathrm{LT}}$ on $T^{-1 / 3}$ which is called the LT plot.

The temperature dependence of Eq. (5) [or of a slightly modified form, the so-called corrected CT (CCT) approach $^{16}$ ] was used for the representation of a large body of experimental vibrational relaxation rates of homonuclear diatomic molecules in monatomic heat bathes (see, e.g., the review Ref. 24 and references cited therein). It should be noted that a temperature dependence which somewhat differs from that of Eq. (5) is predicted for potentials deviating from exponential repulsion. ${ }^{25,26}$

\section{COMMON TRAJECTORY AND SEMICLASSICAL EFFECTIVE MASS APPROXIMATIONS}

In the framework of a quantum treatment of the collision, the effect of rotation of the diatom on the vibrational transition is quite accurately described by the CS approximation which assumes the conservation of the projection of the intrinsic angular momentum of the diatom onto the collision axis. ${ }^{27,28}$ The CT counterpart of this approximation can be readily formulated: it requires the determination of trajectories in the space of two variables, $R=R(t)$ and $\gamma=\gamma(t)$, generated by the equation of motion employing a classical Hamiltonian with the interaction potential $U(R ; \gamma)$. The effective mass (EM) approximation for the determination of common trajectories consists in the introduction of a single "driving mode" $q$ in a localized region of the $R, \gamma$ space where the vibrational transition occurs ${ }^{29}$ (for a more detailed description of the EM approximation see Ref. 30). The driving mode is a specific combination of the coordinate displacements $\Delta R$ and $\Delta \gamma$ that describes the motion along the gradient of the potential $U(R ; \gamma)$ in a small region centered at $R^{*}$ and $\gamma^{*}$. The two-dimensional potential $U\left(R^{*}+\Delta R ; \gamma^{*}\right.$ $+\Delta \gamma)$ is then replaced by a one-dimensional potential $U^{\mathrm{EM}}\left(q ; R^{*}, \gamma^{*}\right)$ "along the gradient" and the reduced mass $\mu$ is changed into the effective mass $\mu^{\text {eff }}$ which depends on $\mu$, the moment of inertia of the diatom $I$, and the contour line $R^{*}=R^{*}\left(\gamma^{*}\right)$ of the potential $U\left(R^{*}, \gamma^{*}\right)$ for a given total energy. Thus the applicability of the EM approach in accounting the effect of rotation of the diatom onto vibrational relaxation is limited by the condition that the rotation angle of the diatom axis with respect to the collision axis during the collision time should be small compared to the angular range of the interaction anisotropy. When this condition is written as $\left|d R^{*} / d \gamma^{*}\right| \ll R^{*}$ (see Ref. 29), the expression for the effective mass $\mu^{\text {eff }}$ assumes the form

$$
\mu^{\mathrm{eff}}\left(\gamma^{*}\right)=\mu /\left(1+\mu\left(d R^{*} / d \gamma^{*}\right)^{2} / I\right) .
$$

Taking, as an example, then interaction of an atom with a homonuclear diatom and adopting a simple parametrization of the contour line,

$$
R^{*}\left(\gamma^{*}\right)=R_{\text {lin }}-\Delta R \cos ^{2} \gamma^{*},
$$

one gets

$$
\mu^{\mathrm{eff}}\left(\gamma^{*}\right)=\mu /\left(1+\frac{\mu(\Delta R)^{2}}{I} \sin ^{2} 2 \gamma^{*}\right) .
$$

We note that the second term in the parentheses is identical with the parameter $Q$ introduced in Ref. 31 in connection with the description of the effectiveness of rotationaltranslational energy transfer on atom-homonuclear diatom collisions, and that Eq. (8) coincides with the expression for the effective mass derived in Ref. 32 if the $\Delta R$ is identified with half of the equilibrium bond distance in the diatom.

Due to the steep $R$ dependence of the interaction, the inverse dependence of the contour lines on the energy is weak in the energy range of interest, so that one can ignore it altogether. Then $\mu^{\text {eff }}$ and $U^{\mathrm{EM}}$ can be parametrized only through $\gamma^{*}\left[\right.$ i.e., $\mu^{\text {eff }}=\mu^{\text {eff }}\left(\gamma^{*}\right)$ and $\left.U^{\mathrm{EM}}=U^{\mathrm{EM}}\left(q, \gamma^{*}\right)\right]$, and the collision time within the EM approach takes the form

$$
\tau^{\mathrm{EM}}\left(E, \gamma^{*}\right)=\int_{q_{t}\left(\gamma^{*}\right)}^{q_{s}} \frac{\sqrt{2 \mu^{\mathrm{eff}}\left(\gamma^{*}\right)} d q}{\sqrt{U^{\mathrm{EM}}\left(q, \gamma^{*}\right)-E}},
$$

where $E$ is the energy of the driving mode. The common trajectory-effective mass (CTEM) transition probability now reads

$$
P_{10}^{\mathrm{CTEM}}\left(E, \gamma^{*}\right)=A^{\mathrm{CTEM}}\left(\gamma^{*}\right) \exp \left(-2 \omega \tau^{\mathrm{EM}}\left(E, \gamma^{*}\right)\right),
$$

and the CTEM rate coefficient is obtained as the thermally averaged probability flux through the surface with the surface element $d S^{*}=2 \pi\left(R^{*}\left(\gamma^{*}\right)\right)^{2} \sin \gamma^{*} d \gamma^{*}$,

$$
\begin{aligned}
k_{10}^{\mathrm{CTEM}}(T)= & \sqrt{\frac{8 k T}{\pi \mu}} \int_{S^{*}} d S^{*} \int_{0}^{\infty} A^{\mathrm{CTEM}}\left(\gamma^{*}\right) \\
& \times \exp \left(-2 \omega \tau^{\mathrm{EM}}\left(E, \gamma^{*}\right)-E / k T\right) \frac{d E}{k T} .
\end{aligned}
$$

This is the final expression for the three-dimensional (3D) CTEM rate coefficient based on a one-dimensional (1D) CTEM transition probability (see also Ref. 29).

The SC modification of the CTEM expression (SCEM) is obtained from Eq. (11) by replacing the CTEM exponential by its SCEM counterpart, ${ }^{16}$

$$
\begin{aligned}
k_{10}^{\mathrm{SCEM}}(T)= & \sqrt{\frac{8 k T}{\pi \mu}} \int_{S^{*}} d S^{*} A^{\mathrm{CTEM}}\left(\gamma^{*}\right) \\
& \times \int_{0}^{\infty} \exp \left(-\frac{2}{\hbar} \int_{E_{i}}^{E_{i}+\hbar \omega} \tau^{\mathrm{EM}}\left(E, \gamma^{*}\right) d E\right. \\
& \left.-E_{i} / k T\right) \frac{d E_{i}}{k T}
\end{aligned}
$$

where $E_{i}$ is the initial energy of the driving mode. The righthand side of Eq. (12) contains a double integral, which 
makes it difficult to conclude on the temperature dependence of the rate coefficient. However, if the interaction anisotropy is small, the double integral in Eq. (12) can be factored into two one-dimensional integrals, one of which carries the main temperature dependence. This is done by writing $\tau^{\mathrm{EM}}\left(E, \gamma^{*}\right)$ as $\tau^{\mathrm{EM}}\left(E, \gamma^{*}\right)=\widetilde{\tau}(E)+\Delta \tau^{\mathrm{EM}}\left(E, \gamma^{*}\right)$ with $\left|\Delta \tau^{\mathrm{EM}}\left(E, \gamma^{*}\right)\right| \ll \widetilde{\tau}(E)$ and determining the energy average in the steepest descent approximation in zero order with respect to the correction $\Delta \tau^{\mathrm{EM}}\left(E, \gamma^{*}\right)$. The zero order steepest descent energy $E_{i}^{*}(T)$ is calculated from the condition that the zero-order exponent in Eq. (12) is minimum which yields an equation for $E_{i}^{*}(T)$, viz,

$$
\widetilde{\tau}\left(E_{i}^{*}\right)-\widetilde{\tau}\left(E_{i}^{*}+\hbar \omega\right)=\hbar / 2 k T .
$$

In the limit $\hbar \omega \ll k T$, Eq. (13) becomes equal to Eq. (4), and $E_{i}^{*}$ coincides with $E^{*}$.

Once $E_{i}^{*}(T)$ is known, the expression for $k_{10}^{\mathrm{SCEM}}(T)$ assumes the form

$$
k_{10}^{\mathrm{SCEM}}(T)=\sqrt{\frac{8 k T}{\pi \mu}} S_{10}^{\mathrm{SCEM}}(T) F_{10}^{\mathrm{SCL}}(T),
$$

where $S_{10}^{\mathrm{SCEM}}(T)$ is the effective cross section,

$$
\begin{aligned}
S_{10}^{\mathrm{SCEM}}(T)= & \int_{S^{*}} d S^{*} A^{\operatorname{CTEM}}\left(\gamma^{*}\right) \\
& \times \exp \left(-\frac{2}{\hbar} \int_{E_{i}^{*}(T)}^{E_{i}^{*}(T)+\hbar \omega} \Delta \tau^{\mathrm{EM}}\left(E, \gamma^{*}\right) d E\right),
\end{aligned}
$$

and $F_{10}^{\mathrm{SCL}}(T)$ represents the SC Landau generalization of the CT expression in Eq. (3),

$$
\begin{aligned}
F_{10}^{\mathrm{SCL}}(T)= & \int_{0}^{\infty} \exp \left(-\frac{2}{\hbar} \int_{E_{i}}^{E_{i}+\hbar \omega} \tilde{\tau}(E) d E-E_{i} / k T\right) \frac{d E_{i}}{k T} \\
= & \frac{1}{k T} \sqrt{\frac{\pi \hbar}{\tilde{\tau}^{\prime}\left(E_{i}^{*}\right)-\tilde{\tau}^{\prime}\left(E_{i}^{*}+\hbar \omega\right)}} \\
& \times \exp \left(-\frac{2}{\hbar} \int_{E_{i}^{*}(T)}^{E_{i}^{*}(T)+\hbar \omega} \tilde{\tau}(E) d E-E_{i}^{*}(T) / k T\right),
\end{aligned}
$$

with $\widetilde{\tau}^{\prime}\left(E_{i}^{*}\right)=\left.(d \widetilde{\tau}(E) / d E)\right|_{E-E_{i}^{*}}$. The exponential factor in Eq. (16) is equal to the exponential factor in Eq. (5) provided that $\widetilde{\tau}(E)=\tau^{\mathrm{LT}}(E)$ and $\hbar \omega / k T \ll 1$. From Eq. (12) one can get lower and upper bounds to $k_{10}^{\mathrm{SCEM}}(T), k_{10, \min }^{\mathrm{SCEM}}(T)$, and $k_{10, \max }^{\mathrm{SCEM}}(T)$ which bracket $k_{10}^{\mathrm{QCEM}}(T)$. This is done by replacing ${ }^{\mathrm{EM}}\left(E, \gamma^{*}\right)$ with its maximal or minimal values with respect to $\gamma^{*}$ and factorizing the double integral. The result reads

$$
\begin{aligned}
k_{10 ; \text { min,max }}^{\mathrm{SCEM}}(T)= & \sqrt{\frac{8 k T}{\pi \mu}} S_{10}^{\mathrm{CTEM}} \\
& \times \int_{0}^{\infty} \exp \left(-\frac{2}{\hbar} \int_{E_{i}}^{E_{i}+\hbar \omega} \tau_{\text {max }, \text { min }}^{\mathrm{EM}}(E) d E\right. \\
& \left.-E_{i} / k T\right) \frac{d E_{i}}{k T}
\end{aligned}
$$

where $\tau_{\max , \min }^{\mathrm{EM}}(E)$ are the maximal or minimal values of $\tau^{\mathrm{EM}}\left(E, \gamma^{*}\right)$ and

$$
S_{10}^{\mathrm{CTEM}}=\int_{S^{*}} A^{\mathrm{CTEM}}\left(\gamma^{*}\right) d S^{*} .
$$

The steepest descent version of Eq. (17) is given by

$$
\begin{aligned}
k_{10 ; \text { min,max }}^{\mathrm{SCEM}}(T)= & \sqrt{\frac{8 k T}{\pi \mu}} S_{10}^{\mathrm{CTEM}} F_{10}^{\mathrm{QCL}}(T) \\
& \times \exp \left(-\frac{2}{\hbar} \int_{E_{i}^{*}}^{E_{i}^{*}+\hbar \omega} \Delta \tau_{\text {max }, \text { min }}^{\mathrm{EM}}(E) d E\right),
\end{aligned}
$$

where $\Delta \tau_{\max , \min }^{\mathrm{EM}}(E)$ is the maximal or minimal values of $\Delta \tau^{\mathrm{EM}}\left(E, \gamma^{*}\right)$.

Equations (12)-(19) are the main result of the Landau generalization of the Landau-Teller treatment for arbitrary interaction potentials $U(R, \gamma)$ within the EM approximation. The latter may become inappropriate if the condition $\left|d R^{*} / d \gamma^{*}\right| \ll R^{*}$ is violated. This can happen, e.g., for collisions of hydrogen halides with rare gas atoms. Actually, it was shown quite a time ago within the semiclassical approach $^{33}$ and recently within accurate VCC CS calculations $^{34,35}$ that for the latter system the EM model fails.

\section{AN EXAMPLE: THE VIBRATIONAL RELAXATION OF $\mathrm{N}_{2}(v=1)$ IN $\mathrm{He}$}

In the following, we choose the vibrational relaxation of $\mathrm{N}_{2}$ in He heat bath,

$$
\mathrm{N}_{2}(v=1 \text {, thermal } j)+\mathrm{He} \rightarrow \mathrm{N}_{2}(v=0 \text {, all } j)+\mathrm{He},
$$

as a simple example for the illustration of the application of the semiclassical-effective mass method described in Sec. III. There are several reasons for choosing this system as an example:

Experimental results exist over a very wide temperature range, from thousands of degrees ${ }^{36}$ down to $70 \mathrm{~K}^{37-39}$ Since the characteristic vibrational temperature of $\mathrm{N}_{2}$ is large $\left(T_{\mathrm{vib}}=\hbar \omega / k=3360 \mathrm{~K}\right)$, quantum effects are expected to manifest themselves within the temperature range of the experiments.

(ii) There exist accurate quantum calculations on the CS level of approximations which showed good agreement with the experimental data. ${ }^{38,39}$

(iii) The low asymmetry of the interaction and the presumably small effect of the molecular rotation result in an only small difference between $k_{10, \text { min }}^{\mathrm{SCEM}}(T)$ and $k_{10, \max }^{\mathrm{SCEM}}(T)$.

Before applying the semiclassical EM approach outlined in Sec. III, we discuss the main features of the interaction potential $U(R, \gamma)$ and of the effective mass $\mu^{\text {eff }}$. Figure 1 shows a logarithmic plot of the ab initio interaction potential (from Fig. 8 of Ref. 38) for two configurations, the linear approach of $\mathrm{He}$ to $\mathrm{N}_{2}(\gamma=0)$ and an approach in T-shaped geometry $(\gamma=\pi / 2)$. The exponential character of the repulsion at high energies is evident, with the logarithmic derivative of the potentials being given by $|d \ln U(R) / d R|$ $=1 / a_{\text {high }}=2.2 / a_{0}=4.15 \AA^{-1} \quad\left(a_{0}\right.$ denotes Bohr's radius $)$. Since the repulsion at higher energies is dominated by ex- 


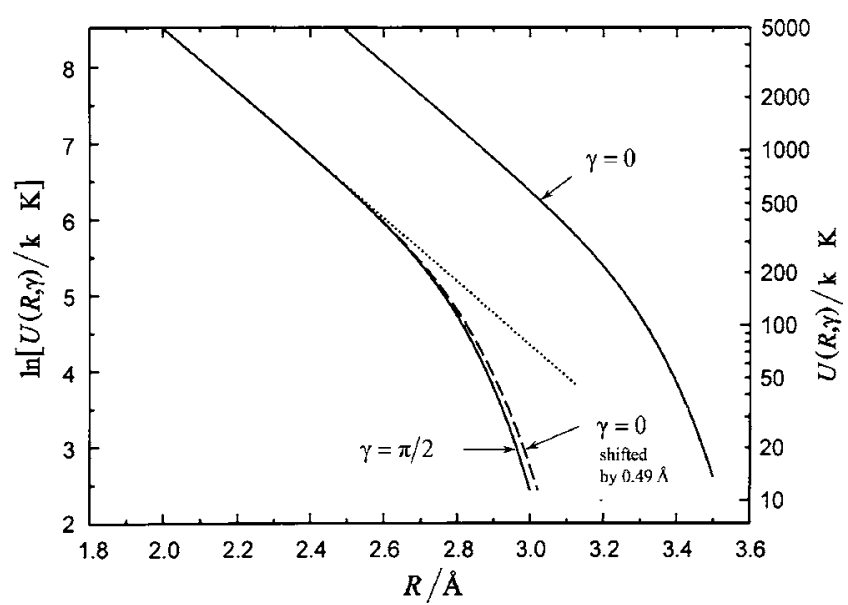

FIG. 1. Interaction potential for the $\mathrm{N}_{2}-\mathrm{He}$ system in collinear $(\gamma=0)$ and T-shaped $(\gamma=\pi / 2)$ configuration, constructed from Fig. 8 of Ref. 38. Shown are also the shifted potential for $\gamma=0$ (dashed line) and an exponential approximation to the high-energy part of the potential (dotted straight line).

change interaction, $U_{\text {exch }}(R)$, it is interesting to compare the above value of $a_{\text {high }}$ with an ab initio estimate from the asymptotic method. ${ }^{40,41}$ Taking into account that the He atom represents a "compact" perturbation (the electronic density in the $\mathrm{He}$ atom with increasing distance from the nucleus decays considerably faster than that in $\mathrm{N}_{2}$ ), the exchange interaction potential at the distance $R$ is proportional to the electron density of the outer molecular orbital (MO) of $\mathrm{N}_{2}$ at this distance. $^{40,41}$ Adopting the known asymptotic form of the outer MO wave function $\psi$ in $\mathrm{N}_{2}$, we get $U_{\text {exch }}(R) \propto|\phi(R)|^{2}$ $\propto R^{2(1 / \hat{a}-1)} \exp \left(-2 \hat{a} R / a_{0}\right)$, where $\hat{a}=\sqrt{2 I_{\mathrm{N}_{2}}}$ and $I_{\mathrm{N}_{2}}$ is the ionization potential of $\mathrm{N}_{2}$ in atomic units. The steepness of the repulsion, the logarithmic derivative of the exchange repulsion $U_{\text {exch }}(R)$ at distance $R$, is equal to

$$
\left|\frac{d \ln U_{\text {exch }}(R)}{d R}\right|=\frac{1}{a_{\text {asym }}}=\frac{2 \hat{a}}{a_{0}}-2 \frac{1 / \hat{a}-1}{R} .
$$

Taking $I_{\mathrm{N}_{2}}=0.572$ a.u. we find $\hat{a}=1.07$ and, at $R$ near to $5 a_{0}$ (see Fig. 1), we get $1 / a_{\text {asym }} \approx 2.17 / a_{0}$ which is very close to $1 / a_{\text {high }}=2.2 / a_{0}$. Note that the second, $R$ dependent, term of the right-hand side of Eq. (21) is only small, indicating that the repulsion is nearly exponential. For this interaction potential, the collision time at high energies for both configurations is equal to the familiar LT expression $\tau_{\text {high }}^{\mathrm{EM}}\left(E, \gamma^{*}\right)_{\gamma^{*}=0, \pi / 2}=\pi a_{\text {high }} / \sqrt{2 E / \mu}$. Note that the effective mass, as defined by Eq. (6), equals $\mu$ in these two configurations, since $\left.\left(d R^{*} / d \gamma^{*}\right)\right|_{\gamma^{*}=0, \pi / 2}$ vanishes due to the symmetry of the interaction.

With decreasing energy the steepness of the interaction increases which is a result of the existence of a potential well. Consequently, the collision time becomes shorter than $\tau_{\text {high }}^{\mathrm{EM}}\left(E, \gamma^{*}\right)$. This is illustrated in Fig. 2 which shows the energy dependence of the ratio $\tau^{\mathrm{EM}}\left(E, \gamma^{*}\right) / \tau_{\text {high }}^{\mathrm{EM}}\left(E, \gamma^{*}\right)$ for $\gamma^{*}=0$ and $\gamma^{*}=\pi / 2$ (dashed lines). Since these two ratios are very close to each other for $\gamma^{*}=0$ and $\pi / 2$, in the following we use the mean value (full line) and assume that it is independent from $\gamma^{*}$.

Adopting the expression (8) for the effective mass, we find $\mu_{\max }^{\text {eff }}=\mu$ and $\mu_{\min }^{\text {eff }}=\mu\left(1-\mu(\Delta R)^{2} / I\right)$. We also see that

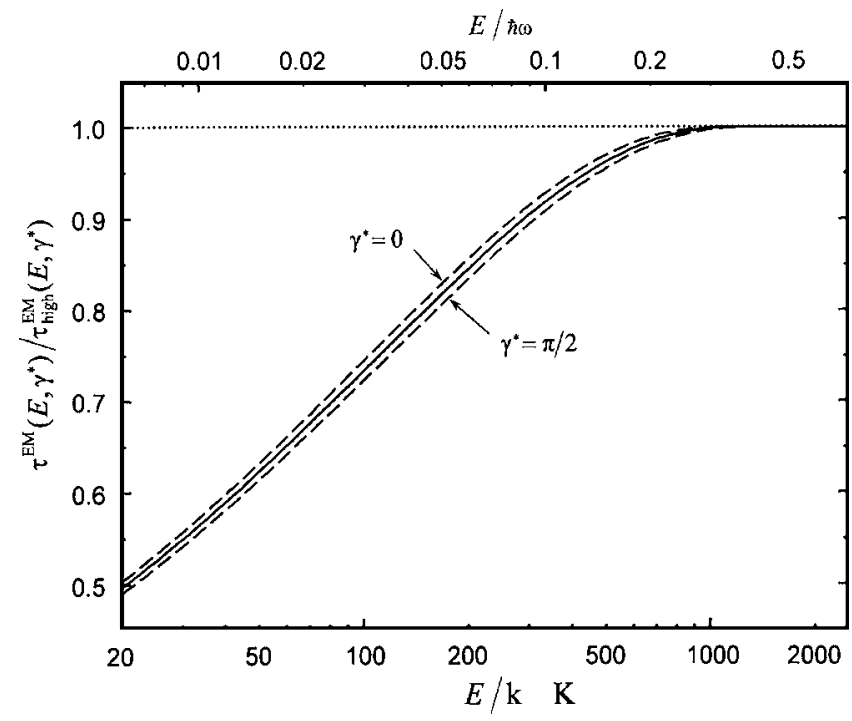

FIG. 2. Ratio of the effective mass collision time $\tau^{\mathrm{EM}}\left(E, \gamma^{*}\right)$ to the highenergy collision time $\tau_{\text {high }}^{\mathrm{EM}}\left(E, \gamma^{*}\right)$ for collinear $\left(\widetilde{a}^{*}=0\right)$ and T-shaped $\left(\widetilde{a}^{*}\right.$ $=\partial / 2$ ) configuration (dashed lines). The full line represents the average value of the ratio used in the present calculations.

the effective mass $\mu^{\text {eff }}$ is virtually independent of the energy, since the two potentials $U(R, 0)$ and $U(R, \pi / 2)$ are nearly superimposed by using a constant value of $\Delta R, \Delta R$ $=0.49 \AA$ (see Fig. 1). Using this values of $\Delta R$ and the moment of inertia of $\mathrm{N}_{2}$, we find $Q \approx 0.1$, i.e., $\mu_{\min }^{\text {eff }}=0.9 \mu$. The small value of the ratio $\left(\mu_{\max }^{\text {eff }}-\mu_{\min }^{\text {eff }}\right) / \mu \approx 0.1$ is the condition for the applicability of the small-anisotropy approximation in Eq. (19). When the values of $\mu_{\max }^{\text {eff }}$ and $\mu_{\min }^{\text {eff }}$ are known, the respective values of $\tau_{\max }^{\mathrm{EM}}$ and $\tau_{\min }^{\mathrm{EM}}$ can be recovered from the relation $\tau^{\mathrm{EM}} \propto \sqrt{\mu^{\text {eff }}}$.

Apart of a constant factor, the given information is sufficient for the calculation of $k_{10, \sin }^{\mathrm{SCEM}}(T)$ and $k_{10, \max }^{\mathrm{SCEM}}(T)$. The rate coefficients $k_{10, \text { min }}^{\mathrm{SCEM}}(T)$ and $k_{10, \text { max }}^{\mathrm{S} \mathrm{CEM}}(T)$, such as calculated from Eq. (17) and fitted to the experimental data points and to accurate CS results by a common shift along the abscissa axis (numerical fitting of $S_{10}^{\text {CTEM }}$ ), are shown in the LT plot of Fig. 3 as two boundaries of the region covered by dots. The experimental data points (and the accurate CS results) from Refs. 38 and 39 are well enclosed in this region. Note that this fitting provides a good representation of the temperature dependence of the rate coefficient over eight orders of magnitude.

For the considered example, one may well characterize the various contributions to the rate coefficient. This is also illustrated in Fig. 3. In addition to $k_{10, \min }^{\mathrm{SCEM}}(T)$ and $k_{10, \max }^{\mathrm{SCEM}}(T)$, we show rate coefficients for a purely exponential LT interaction $U \propto \exp \left(-R / a_{\text {high }}\right)$ such as employed in the LT model: the common trajectory LT rate coefficient $k_{10}^{\mathrm{CTLT}}(T)$ (lower dashed curve), the corrected common trajectory LT rate coefficient $k_{10}^{\mathrm{CCTLT}}(T)$ (upper dashed curve), and the semiclassical LT rate coefficient $k_{10}^{\mathrm{SCLT}}(T)$ (middle dashed curve). We notice that quantum effects are already visible at $T$ $=2000 \mathrm{~K}$, and that the CCT rate coefficient provides a reasonable approximation to SCLT rate coefficient down to $T$ $=500 \mathrm{~K}$. The combined effect of the attraction and the rota- 


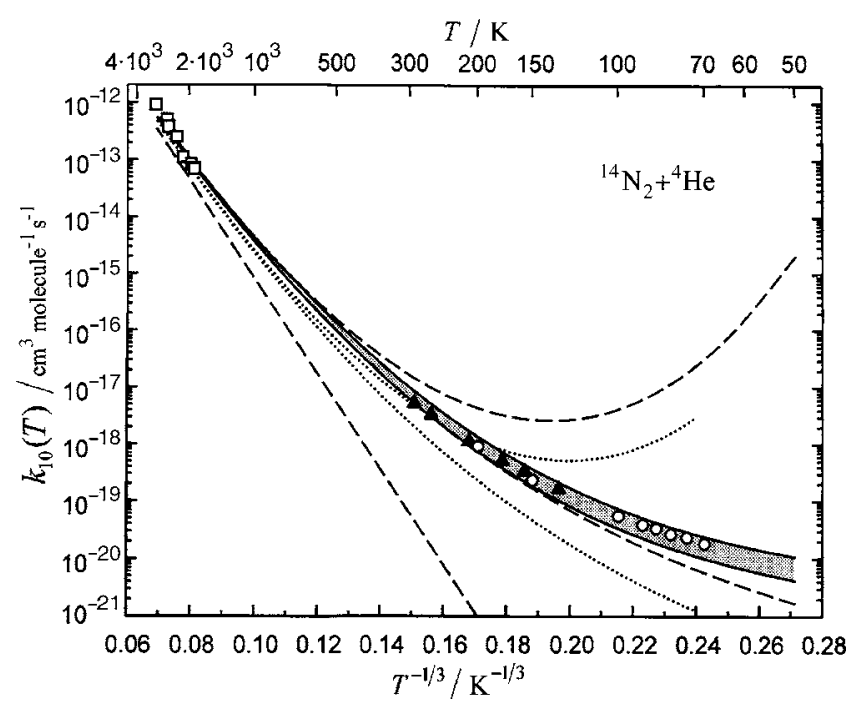

FIG. 3. Rate coefficients $k_{10}(T)$ for ${ }^{14} \mathrm{~N}_{2}$ relaxation in ${ }^{4} \mathrm{He}$, normalized by fitting the preexponential factor to the high-temperature relaxation data from Ref. 36 (open squares). The full lines correspond to $k_{10, \min }^{\mathrm{SCEM}}(T)$ and $k_{10, \max }^{\mathrm{SCEM}}(T)$ and the dotted area between them represents the region of the accurate $k_{10}^{\mathrm{SCEM}}(T)$. The dashed lines, in increasing order, represent the rate coefficients for the exponential repulsion with the high-energy value of the potential steepness: $k_{10}^{\mathrm{CTLT}}(T)$ (i.e., the linear Landau-Teller plot), $k_{10}^{\mathrm{SCLT}}(T)$, and $k_{10}^{\text {CCTLT }}(T)$. The experimental data points at low temperatures (which agree with CS calculations) are presented by filled triangles (Ref. 37) and open circles (Ref. 38). The dotted curves from Ref. 24 represent $k_{10}^{\text {CTLT }}(T)$ and $k_{10}^{\mathrm{SCLT}}(T)$ employing a slightly lower steepness parameter (see text).

tional enhancement clearly shows up below $200 \mathrm{~K}$ (comparing the dashed SCLT curve with the dotted region).

The difference between $k_{10}^{\mathrm{SCLT}}(T)$ and $k_{10, \mathrm{~min}}^{\mathrm{SCEM}}(T)$, as noted in Ref. 37, is due to the attractive well which makes the potential steeper than its high-energy part. This difference, however, is only small if one compares the collision time at $T=50 \mathrm{~K}$ with its high-energy counterpart. The explanation of an only small effect of the attraction on the relaxation rate at low temperatures can be found in the observation that $\widetilde{\tau}(E)$ enters into the expression for the rate through the integral over a broad energy range of a width of the value of $\hbar \omega$ (the quantum effect), and that the main contribution to the rate comes from the range of energies between $E_{i}^{*}$ and $E_{i}^{*}+\hbar \omega$ provided that the steepest descent method is applicable, i.e., when $E_{i}^{*}$ noticeable exceeds the thermal energy $E_{T}=k T$. Figure 4 shows graphs of $E_{i}^{*}, E_{i}^{*}+\hbar \omega, E^{*}$, and $E_{T}$ as functions of the temperature. At lower temperatures (say, below $100 \mathrm{~K}$ ), the condition $E_{i}^{*} \gg k T$ is not fulfilled, and this is reflected in the progressively weaker temperature dependence of the rate coefficient (see Fig. 3).

The sensitivity of the calculated rate coefficient to the key parameter, the steepness of the potential, is illustrated by the dotted lines in Fig. 3. They are drawn for $k_{10}^{\mathrm{CCTLT}}(T)$ and $k_{10}^{\mathrm{SCLT}}(T)$ with $1 / a_{\text {high }}=3.8 \AA^{-1}$, the value adopted in Ref. 24 for the description of the experimental high-temperature data $^{36}$ and data point at $T=291 \mathrm{~K}$ from Ref. 37 . We see, however, that, for this value of the steepness parameter, $k_{10}^{\text {CCTLT }}(T)$ near to $T=300 \mathrm{~K}$ already deviates noticeably from $k_{10}^{\mathrm{SCLT}}(T)$.

In the following we also illustrate the isotope effect of energy transfer. A change of the masses of the colliding species can lead to a considerable change in the vibrational re-

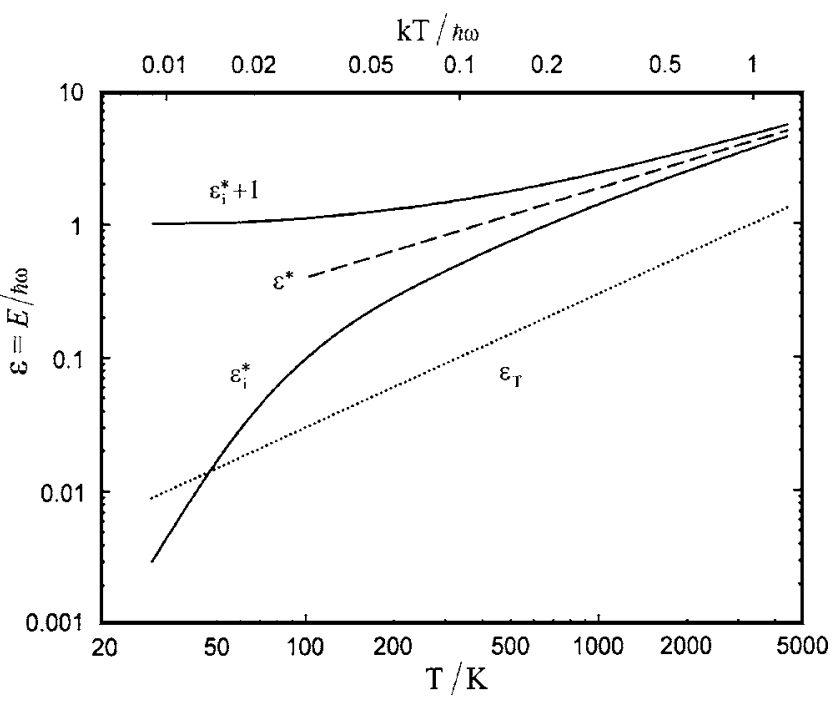

FIG. 4. Reduced steepest descent values of the initial $\varepsilon_{i}^{*}$ and final $\varepsilon_{i}^{*}+1$ energies (full lines), the steepest descent Landau-Teller energy $\varepsilon^{*}$ (dashed line), and the mean thermal energy $\varepsilon_{T}=k T / \hbar \omega$ (dotted line) for $\mathrm{N}_{2}-\mathrm{He}$ collisions.

laxation rate. This change originates from a change in the reduced mass of the partners and from a change in the vibrational frequency of the diatom. We compare the systems ${ }^{14} \mathrm{~N}_{2}+{ }^{4} \mathrm{He}$ and ${ }^{15} \mathrm{~N}_{2}+{ }^{3} \mathrm{He}$, for which both experimental and theoretical results (on the CS level) are available. ${ }^{38}$ If one would assume that the isotope effect is exclusively due to the change in the exponential term in the integrand in Eq. (12), the dotted region bounded by $k_{10 \text {, min }}^{\mathrm{SCEM}}\left(T,{ }^{14} \mathrm{~N}_{2}+{ }^{4} \mathrm{He}\right)$ and $k_{10 \text {,max }}^{\mathrm{SCEM}}\left(T,{ }^{14} \mathrm{~N}_{2}+{ }^{4} \mathrm{He}\right)$ curves in Fig. 5 would change into the region bounded by the two dashed lines. This region does not enclose the experimental data points for the ${ }^{15} \mathrm{~N}_{2}+{ }^{3} \mathrm{He}$ system in the same manner as it does for the ${ }^{14} \mathrm{~N}_{2}+{ }^{4} \mathrm{He}$ system. One may, therefore, suspect that this observation is due to the neglect of the dependence of the preexponential factor on the

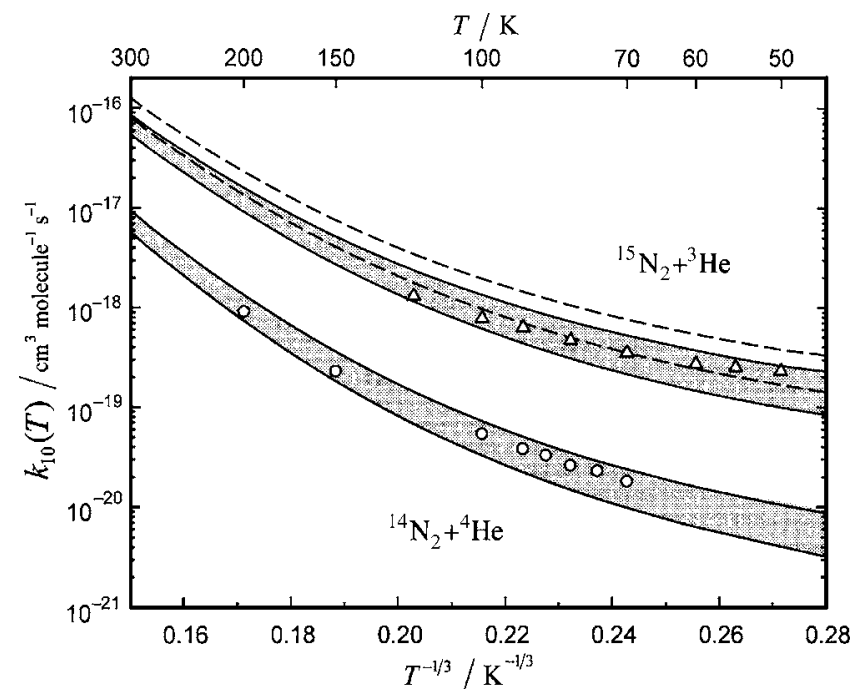

FIG. 5. Rate coefficients $k_{10}(T)$ for ${ }^{14} \mathrm{~N}_{2}$ relaxation in ${ }^{4} \mathrm{He}$ (lower points) and ${ }^{15} \mathrm{~N}_{2}$ relaxation in ${ }^{3} \mathrm{He}$ (upper points). The dotted regions correspond to the range between $k_{10, \sin }^{\mathrm{SCEM}}(T)$ and $k_{10, \max }^{\mathrm{SCEM}}(T)$. The region between the dashed curves for the ${ }^{15} \mathrm{~N}_{2}+{ }^{3} \mathrm{He}$ system corresponds to the range calculated with the same preexponential factor as for ${ }^{14} \mathrm{~N}_{2}+{ }^{4} \mathrm{He}$ collisions. The symbols correspond to the experimental results from Ref. 38, Table II. 
reduced mass which changes quite significantly when passing from ${ }^{4} \mathrm{He}$ to ${ }^{3} \mathrm{He}$. Indeed, a reference to the simple breathing sphere model reveals that the preexponential factor scales as $\mu^{3 / 2}$ (see Ref. 29). When this effect is taken into account, this region slightly lowers and becomes the dotted region for ${ }^{15} \mathrm{~N}_{2}+{ }^{3} \mathrm{He}$ collisions in Fig. 5 which well covers the experimental points.

\section{SUMMARIZING REMARKS}

The temperature dependence of the vibrational relaxation rate coefficient of diatomic molecules, at bath gas temperatures comparable or higher than the characteristic vibrational temperature $T_{\text {vib }}=\hbar \omega / k$, is usually described by the Landau-Teller formula with simple quantum corrections that ensure detailed balance between up and down transitions. The additional assumption of collinear collision dynamics in the LT formula does not readily reveal itself in collisions of homonuclear diatoms with light atoms, because the vibrational transition occurs in a localized region near to the turning point of the relative motion where the diatom has no time for substantial rotation. The assumption of an exponential repulsion between the diatom and heat bath atoms appears compatible with the picture that at high temperatures the exchange interaction is of dominant importance. With temperature falling to values below $T_{\mathrm{vib}}$, the dynamical picture becomes more complicated: (i) quantum effects, which are related to the release of a large energy quantum $\hbar \omega$ into the thermal bath of temperature $T$, become important; (ii) due to the existence of the attractive well, the interaction potential is sampled in a region where it noticeably deviates from exponential form; and (iii) because of the increase in the characteristic collision time, the rotation of the diatom begins to play a role. At still lower temperatures, when the thermal energy $k T$ becomes comparable to or lower than the well depth $D$ of the potential, an atom-diatom transient complex can be formed which is the result of an energy exchange between the relative translation of the partners and the rotation of the diatom. Under this condition, multiple encounters between the partners in the region of the repulsive wall of the potential are possible. Thus the picture of a direct vibrationally inelastic collision becomes invalid. If, in addition, the conditions of quasiclassical relative motion and rotation of the diatom are not fulfilled, one has to resort to the solution of the full quantum-scattering problem (for a recent review see Ref. 42).

For diatomic molecules of low asymmetry (such as homonuclear molecules) colliding with light rare gas atoms, there exists a wide temperature range $D / k<T<\hbar \omega / k$ within which the complex-forming mechanism can be disregarded, but all of the features [(i)-(iii)] mentioned above are of importance. This is the case for $\mathrm{N}_{2}-\mathrm{He}$ collisions over the temperature range of $100-3000 \mathrm{~K}$. Within this range, the rotational motion of $\mathrm{N}_{2}$ and the relative motion of $\mathrm{N}_{2}-\mathrm{He}$ are quasiclassical in the WKB sense, and the only quantum effect, which is felt by the bath gas, is the large quantum change in its energy as a result of the inelastic event. The semiclassical description of the latter feature is possible within the Landau method ${ }^{18}$ of calculating transition matrix elements and expressing them through the classical collision time introduced by Landau and Teller. ${ }^{7}$ In this way it is possible to generalize the basic Landau-Teller treatment to temperatures substantially below than $T_{\text {vib }}$.

Once one recognizes that the main temperature dependence of $k_{10}$ comes from the Landau exponent and if one aims at a description and analysis of this temperature dependence rather than at a calculation of the absolute value of $k_{10}$, it becomes possible to formulate a simple quasiclassical approach. We have illustrated this approach using $\mathrm{N}_{2}-\mathrm{He}$ collisions as an example. On the basis of interaction potentials from Ref. 38 we obtained the following effects which all lead to curvatures of the LT plot (see Fig. 3) in the region below $300 \mathrm{~K}$ and which disappear at the high-temperature side of the plot:

(a) a large quantum effect of the energy release and the early breakdown of the first quantum correction to the common trajectory approach;

(b) a noticeable effect of the deviation of the potential from its high-energy exponential form; and

(c) a noticeable effect of rotational enhancement of the vibrational transition rate.

Our semiclassical extension of the Landau-Teller model is based on the extension of the common trajectory approximation to the Landau semiclassical treatment, the generalization of the collinear collision model to three dimensions in the framework of effective mass approach, and the incorporation of a realistic interaction potential through the LandauTeller collision time. For the $\mathrm{N}_{2}-\mathrm{He}$ example, our analytical formulas describe the temperature dependence of $k_{10}$ over eight orders of magnitude, while the absolute value of $k_{10}$ is recovered by fitting a single parameter only, the preexponential factor in the expression for the rate. Our analysis shows the importance of various factors, which were not included in the original LT model (we did not consider the range where transient atom-diatom complexes are formed, that is where an upturn of the rate coefficient is expected, i.e., at $T$ below $30-40 \mathrm{~K}$ for $\mathrm{N}_{2}-\mathrm{He}$ ). The analysis presented suggests an interpretation of the quantum effects in terms of classical quantities, such as a collision time an effective mass. Compared to the quantum coupled-states calculations, the present treatment provides considerable computational simplifications such that its use for more complicated situations appears promising. It can be helpful, e.g., in the isolated binary collision concept of the vibrational relaxation of molecules in condensed media where quantum calculations are most difficult and Landau-Teller-type approaches are widely used. ${ }^{11-13}$ In terms of accuracy, our analytical treatment cannot compete, of course, with quantum scattering calculations. However, we agree with the recent statement from Ref. 43 that "classical mechanics provides an intuitive picture to understand the underlying mechanism that may be difficult to extract from quantum calculations." ${ }^{43}$ In this spirit, based on our analytical approach, we conclude that the main physical quantity which determines the temperature dependence of the vibrational relaxation rate coefficient in the classical and quantum regimes is the classical LT collision time. 
The VCC-CS calculations, that provide a good approximation to the accurate VCC CC approach, become very heavy for collisions of polyatomic species. On the other hand, if VCC-CS calculations are performed in the basis of adiabatic rovibronic functions of collision partners, they can benefit from the well-developed computational codes for the adiabatic channel (AC) potentials. At low collision energies, such as those of astrophysical interest, the nonadiabatic coupling between adiabatic channel states is expected to be weak. Then the VCC part of the problem can be handled within the perturbation approach, similar to that described in this paper. Thus, the combination of the adiabatic channel approach, as developed in the theory of the complex formation and the $\mathrm{SC}$ nonadiabatic ( $\mathrm{SCN}$ ) theory, can be a proper avenue for attacking more complicated cases than atomdiatom collisions. In the context of the latter, the effective mass approximation described in Sec. III appears as to be a simplification of the more general ACSCN method.

\section{ACKNOWLEDGMENT}

One of the authors (I.L.) acknowledges financial support provided through the European Community's Human Potential Program under Contract No. MCRTN 512302 ("Molecular Universe").

${ }^{1}$ D. C. Clary, in Supercomputer Algorithms for Reactivity, Dynamics and Kinetics of Small Molecules, NATO Advanced Studies Institute, Series C: Matheratical and Physical Sciences, edited by A. Lagana (Kluwer, Dordrecht, 1989), Vol. 277.

${ }^{2}$ A. Faure, P. Valiron, M. Wernli, L. Wiesenfeld, C. Rist, J. Noga, and J. Tannyson, J. Chem. Phys. 122, 221102 (2005).

${ }^{3}$ A. Faure, L. Wiesenfeld, M. Wernli, and P. Valiron, J. Chem. Phys. 123, 104309 (2005).

${ }^{4}$ M. S. Child, Semiclassical Mechanics with Molecular Applications (Clarendon, Oxford, 1991).

${ }^{5}$ G. D. Billing, The Quantum Classical Theory (Oxford University Press, Oxford, 2003).

${ }^{6}$ R. Zwanzig, J. Chem. Phys. 34, 1931 (1961).

${ }^{7}$ L. D. Landau and E. Teller, Phys. Z. Sowjetunion 10, 34 (1936).

${ }^{8}$ S. A. Egorov and J. L. Skinner, Chem. Phys. Lett. 283, 469 (1998).

${ }^{9}$ S. A. Egorov, E. Rabinim, and B. J. Berne, J. Phys. Chem. B 103, 10978 (1999).

${ }^{10}$ G. Käb, Phys. Rev. E 66, 046117 (2002).

${ }^{11}$ G. Käb, J. Phys. Chem. A 108, 8866 (2004).
${ }^{12}$ A. A. Neufeld, J. Chem. Phys. 119, 2488 (2003).

${ }^{13}$ A. A. Neufeld, D. Schwarzer, J. Schröder, and J. Troe, J. Chem. Phys. 119, 2502 (2003)

${ }^{14}$ Q. Shi and E. Geva, J. Phys. Chem. A 107, 9059 (2003).

${ }^{15}$ Q. Shi and E. Geva, J. Phys. Chem. A 107, 9070 (2003).

${ }^{16}$ E. Nikitin and J. Troe, Phys. Chem. Chem. Phys. 8, 2012 (2006).

${ }^{17}$ L. D. Landau and E. M. Lifshitz, Quantum Mechanics (Pergamon, Oxford, 1977).

${ }^{18}$ L. D. Landau, Phys. Z. Sowjetunion 2, 46 (1932).

${ }^{19}$ E. E. Nikitin and L. P. Pitaevski, Phys. Rev. A 49, 695 (1994).

${ }^{20}$ M. Baer, G. Drolshagen, and J. P. Toennies, J. Chem. Phys. 73, 1690 (1980).

${ }^{21}$ G. Drolshagen, J. P. Toennies, and M. Baer, Chem. Phys. Lett. 102, 354 (1983).

${ }^{22}$ E. P. Gordeev and S. Ya. Umanskii, Khim. Fiz. 3, 649 (1984).

${ }^{23}$ Y. Karni and E. E. Nikitin, Chem. Phys. 191, 235 (1995).

${ }^{24}$ E. E. Nikitin, A. I. Osipov, and S. Ya. Umanskii, Khimiya Plazmy (Energoatomizdat, Moscow, 1989), Vol. 15, p. 3.

${ }^{25}$ D. Schwarzer and M. Teubner, J. Chem. Phys. 116, 5680 (2002).

${ }^{26}$ M. Teubner, Phys. Rev. E 65, 031204 (2002).

${ }^{27}$ H. Rabitz, in Dynamics of Molecular Collisions, edited by W. H. Miller (Plenum, New York, 1976), Pt. A.

${ }^{28}$ F. A. Gianturco, The Transfer of Molecular Energies by Collisions (Springer, Berlin, 1979).

${ }^{29}$ E. E. Nikitin, Theory of Elementary Atomic and Molecular Processes in Gases (Clarendon, Oxford, 1974).

${ }^{30}$ T. D. Sewell, S. Nordholm, and A. Miklavć, J. Chem. Phys. 99, 2567 (1993).

${ }^{31}$ F. A. Gianturco and J. P. Toennies, J. Chem. Soc., Faraday Trans. 87, 31 (1991).

${ }^{32}$ A. Miklavc, N. Markovic, G. Nyman, V. Harb, and S. Nordholm, J. Chem. Phys. 97, 3348 (1992).

${ }^{33}$ M. Ya. Ovchinnikova, Chem. Phys. 93, 101 (1985).

${ }^{34}$ R. V. Krems, N. Marković, A. A. Buchachenko, and S. Nordholm, J. Chem. Phys. 114, 1249 (2000).

${ }^{35}$ R. V. Krems, A. A. Buchachenko, N. Marković, and S. Nordholm, J. Chem. Phys. 117, 166 (2002).

${ }^{36}$ D. R. White, J. Chem. Phys. 48, 525 (1968)

${ }^{37}$ M. M. Maricq, E. A. Gregory, C. T. Wickham-Jones, D. J. Cartwright, and C. J. S. M. Simpson, Chem. Phys. 75, 347 (1983).

${ }^{38}$ J. P. Reid, A. J. Thakkar, P. W. Barnes, E. F. Archibong, H. M. Quiney, and C. J. S. M. Simpson, J. Chem. Phys. 107, 2329 (1997).

${ }^{39}$ J. P. Reid, C. J. S. M. Simpson, and H. M. Quiney, Chem. Phys. Lett. 256, 531 (1996).

${ }^{40}$ E. E. Nikitin and S. Ya. Umanskii, Theory of Slow Atomic Collisions (Springer, Berlin, 1984).

${ }^{41}$ K. T. Tang, J. P. Toennies, and C. L. Yiu, Int. Rev. Phys. Chem. 17, 363 (1998).

${ }^{42}$ R. V. Krems, Int. Rev. Phys. Chem. 24, 99 (2005).

${ }^{43}$ A. Faure and L. Wiesenfeld, J. Chem. Phys. 121, 6771 (2004) 